УДК 681.04

Ю.Д. Поліський, к.т.н., polissky477@gmail.com

НДІ автоматизації чорної металургії, м. Дніпро

\title{
РЕАЛІЗАЦІЯ ДЕЯКИХ ПРОБЛЕМНИХ ОПЕРАЦІЙ У СИСТЕМАХ ЗАЛИШКОВИХ КЛАСІВ
}

В роботі досліджені системи залишкових класів з попарно взаємно простими модулями i системи залишкових класів з усіма парними модулями з можливістю реалізації базових проблемних операцій визначення приналежності числа до даної половині діапазону і порівняння чисел.

Ключові слова: залишкові класи, проблемні операчіï, модулі, порівняння чисел.

Systems of residual classes with pairwise mutually simple modules and systems of residual classes with all even modules are investigated with the possibility of implementing basic problem operations for determining the number of a given half of the range and comparing numbers.

Keywords: residual classes, problem operations, modules, comparison of numbers.

\section{Постановка проблеми}

До обчислювальних структур постійно пред'являються вимоги підвищення швидкодії. Застосування системи залишкових класів (СЗК) з попарно взаємно простими модулями дозволяє підвищити продуктивність таких систем за рахунок природного розпаралелювання обробки даних. Останнім часом для реалізації деяких проблемних операцій запропоновані рішення [1], в яких поряд 3 використанням систем попарно взаємно простих модулів застосовуються також і системи, в яких модулі не $\epsilon$ взаємно простими, зокрема - всі парні. При значних перевагах СЗК застосування цих систем наштовхується на певні труднощі.

Аналіз останніх досліджень і публікацій

Переваги СЗК детально викладені в [2,3,4]. Однак виникають складності [5] при реалізації проблемних базових операцій визначення приналежності числа до даної половині діапазону і порівняння чисел.

\section{Формулювання мети дослідження}

Метою дослідження $\epsilon$ аналітичний розгляд СЗК з попарно взаємно простими модулями і СЗК з усіма парними модулями для реалізації базових проблемних операцій визначення приналежності числа до даної половині діапазону і порівняння чисел.

\section{Виклад основного матеріалу}

Під СЗК [6] розуміють систему числення, в якій довільне число $N$ представляється у вигляді набору найменших невід'ємних залишків по модулях $m_{1}, m_{2}, \ldots, m_{n}$, тобто $N=\left(\alpha_{1}, \alpha_{2}, \ldots, \alpha_{n}\right)$. Тут $\alpha_{i}=N\left(\bmod m_{i}\right)$. При цьому, якщо числа $m_{i}$ попарно взаємно прості, то такому представленню відповідає тільки одне число $N$ діапазону $[0, M)$, де $M=m_{1} m_{2} \ldots m_{n}$.

Якщо системою модулів поліадичного коду також $є$ система $m_{1}, m_{2}, \ldots, m_{n}$, число $N$ в поліадичному коді представляється у вигляді

$$
N=\pi_{1}+\pi_{2} m_{1}+\ldots+\pi_{i} m_{1} m_{2} \ldots m_{i-1}+\ldots+\pi_{n-1} m_{1} m_{2} \ldots m_{n-2}+\pi_{n} m_{1} m_{2} \ldots m_{n-1},
$$

де $0 \leq \pi_{i} \leq m_{i}-1$.

Покажемо, що таке представлення є єдиним.

Нехай $\widetilde{N}=\widetilde{N}_{1}+\widetilde{N}_{2}$, де $\widetilde{N}_{1}=\pi_{1}+\pi_{2} m_{1}+\ldots+\pi_{i-1} m_{1} m_{2} \ldots m_{i-2}, \quad \tilde{N}_{2}=\pi_{i} m_{1} m_{2} \ldots m_{i-1}$.

Нехай число $\tilde{N}_{1}$ збільшилося на величину $\Delta$. Для того, щоб представлення числа $N$ в поліадичному коді при цьому не змінилося, необхідно, щоб число $\tilde{N}_{2}$ зменшилося на ту ж величину $\Delta$. 
Нехай в результаті збільшення числа $\tilde{N}_{1}$ отримано найбільше значення $\widetilde{N}_{1, \max }=m_{1} m_{2} \ldots m_{i-1}-1$, яке досягається при $\pi_{t}=m_{t}-1, t=1,2, \ldots,(i-1)$.

Нехай в результаті зменшення числа $\tilde{N}_{2}$ отримано найменше значення $\tilde{N}_{2, \min }=m_{1} m_{2} \ldots m_{i-1}$, яке досягається при $\pi_{i}=1$.

Оскільки $\tilde{N}_{1, \max }<\tilde{N}_{2, \min }$, наведене вище представлення числа $N$ в поліадичному коді є єдиним.

Однією з ключових складних операцій СЗК є операція визначення приналежності числа до першої $R 1$ або другої $R 2$ половині діапазону.

В роботі [7] доведено, що при представленні числа $N$ в поліадичному коді, яке для попарно взаємно простих чисел $m_{i}$ є єдиним, критерієм приналежності числа до даної половині діапазону служить значення $\pi_{n}$

$$
N \in\left\{\begin{array}{l}
R 1, \quad 0 \leq \pi_{n} \leq \frac{m_{n}}{2}-1 \\
R 2, \frac{m_{n}}{2} \leq \pi_{n} \leq m_{n}-1
\end{array}\right.
$$

Знання про приналежність числа до даної половині діапазону дозволяє вирішити задачу порівняння чисел.

Нехай $N_{1}=\left(\alpha_{1}, \alpha_{2}, \ldots, \alpha_{n-j}\right)$ і $N_{2}=\left(\beta_{1}, \beta_{2}, \ldots, \beta_{n-j}\right)$ - порівнювані числа, $N_{1} \neq N_{2}$, та $\Delta=N_{1}-N_{2}$ - різниця чисел $N_{1}$ і $N_{2}$.

Необхідно визначити результат

$$
\mathfrak{R}=\left\{\begin{array}{l}
\mathfrak{R}_{1}, N_{1}>N_{2} \\
\mathfrak{R}_{2}, N_{1}<N_{2}
\end{array}\right.
$$

Порівняння чисел $N_{1}$ і $N_{2}$ виконується відповідно до алгоритму

$$
\mathfrak{R}_{1}=\left\{\begin{array}{c}
\left.N_{1}>N_{2},\left(N_{1} \in R 2 \bigcap N_{2} \in R 1\right) \bigcup\left(N_{1}, N_{2} \in R 1 \bigcup N_{1}, N_{2} \in R 2\right) \bigcap \Delta \in R 1\right) \\
\left.N_{1}<N_{2}, N_{1} \in R 1 \cap N_{2} \in R 2 \bigcup\left(N_{1}, N_{2} \in R 1 \bigcup N_{1}, N_{2} \in R 2\right) \bigcap \Delta \in R 2\right)
\end{array}\right.
$$

Ефективний алгоритм порівняння чисел, не пов'язаний з визначенням належності числа до даної половині діапазону [8], полягає в наступному.

Якщо після $(j-1)$-й ітерації $(j=1,2, \ldots, n-1)$ результат порівняння не отримано, виконується $j$-я ітерація для діапазону $\left[0, M^{j-1}\right)$, де $M^{j-1}=m_{1} m_{2} \ldots m_{n-(j-1)}$.

$$
\begin{aligned}
& \tilde{\alpha}_{i}^{j}=\left(\widetilde{\alpha}_{i}^{j-1}-\widetilde{\alpha}_{n-(j-1)}^{j-1}\right)\left(\bmod m_{i}\right), i=1,2, \ldots, n-(j-1), \\
& \tilde{\beta}_{i}^{j}=\left(\tilde{\beta}_{i}^{j-1}-\tilde{\beta}_{n-(j-1)}^{j-1}\right)\left(\bmod m_{i}\right), i=1,2, \ldots, n-(j-1) .
\end{aligned}
$$

Нехай

$$
\begin{gathered}
\tilde{N}_{1}^{j}=\left(\tilde{\alpha}_{1}^{j}, \tilde{\alpha}_{2}^{j}, \ldots, \tilde{\alpha}_{n-j}^{j}\right), \\
\tilde{N}_{2}^{j}=\left(\tilde{\beta}_{1}^{j}, \tilde{\beta}_{2}^{j}, \ldots, \tilde{\beta}_{n-j}^{j}\right) . \\
\mathfrak{R}=\left\{\begin{array}{c}
N_{1}>N_{2},\left(\tilde{N}_{1}^{j}=\tilde{N}_{2}^{j}\right) \cap\left(\tilde{\alpha}_{n}^{j-1}>\tilde{\beta}_{n}^{j-1}\right) \\
N_{1}<N_{2},\left(\tilde{N}_{1}^{j}=\tilde{N}_{2}^{j}\right) \cap\left(\tilde{\alpha}_{n}^{j-1} \tilde{\beta}_{n}^{j-1}\right)
\end{array}\right.
\end{gathered}
$$


Якщо $\tilde{N}_{1}^{j} \neq \tilde{N}_{2}^{j}, \quad$ виконується $(j+1)$-я ітерація для діапазону $\left[0, M^{j}\right)$, де $M^{j}=m_{1} m_{2} \ldots m_{n-j}$. Для цього приймаємо $\tilde{N}_{1}^{1, j}=\frac{\tilde{N}_{1}^{j}}{m^{n-(j-1)}} \quad$ та $\quad \tilde{N}_{2}{ }^{1, j}=\frac{\tilde{N}_{2}^{j}}{m^{n-(j-1)}}$ в якості порівнюваних чисел.

Якщо результат порівняння не отримано до $(n-1)$-й ітерації, то після ії виконання

$$
\mathfrak{R}=\left\{\begin{array}{l}
N_{1}>N_{2},\left(\left(\tilde{N}_{1}^{1, n-1}=\tilde{N}_{2}^{1, n-1}\right) \cap\left(\tilde{\alpha}_{1}^{1, n-2}>\tilde{\beta}_{1}^{1, n-2}\right)\right) \cup \\
\cup\left(\left(\tilde{N}_{1}^{1, n-1} \neq \tilde{N}_{2}^{1, n-1}\right) \cap\left(\tilde{\alpha}_{1}^{1, n-1}>\tilde{\beta}_{1}^{1, n-1}\right)\right) \\
N_{1}<N_{2},\left(\left(\tilde{N}_{1}^{1, n-1}=\tilde{N}_{2}^{1, n-1}\right) \cap\left(\tilde{\alpha}_{1}^{1, n-2}<\tilde{\beta}_{1}^{1, n-2}\right)\right) \cup \\
\cup\left(\left(\tilde{N}_{1}^{1, n-1} \neq \tilde{N}_{2}^{1, n-1}\right) \cap\left(\tilde{\alpha}_{1}^{1, n-1}<\tilde{\beta}_{1}^{1, n-1}\right)\right)
\end{array}\right.
$$

В СЗК з використанням систем модулів, які не $є$ взаємно простими, зокрема -3 усіма парними модулями, представленню $N=\left(\alpha_{1}, \alpha_{2}, \ldots, \alpha_{n}\right)$ відповідають кілька чисел діапазону $[0, M)$.

Величина $M$, що дорівнює добутку $m_{1}, m_{2}, \ldots, m_{n}$ в системі взаємно простих модулів $m_{i}, \epsilon$, по суті, найменшим спільним кратним цих модулів, тобто $M=m_{1} m_{2} \ldots m_{n}=\left\langle m_{1}, m_{2}, \ldots, m_{n}\right\rangle$. Якщо поняття величини $M$ як найменшого спільного кратного поширити на систему модулів, які не $\epsilon$ взаємно простими, то і в цьому випадку кожному числу з $[0, \hat{M})$ відповідатиме єдиний набір $\hat{N}=\left(\hat{\alpha}_{1}, \hat{\alpha}_{2}, \ldots, \hat{\alpha}_{n}\right)$. При такому узагальненні число $N$ в поліадичному коді для тієї ж системи модулів, які не є взаємно простими, представляється у вигляді

$$
\hat{N}=\pi_{1}+\pi_{2}\left\langle m_{1}\right\rangle+\ldots+\pi_{i}\left\langle m_{1}, m_{2}, \ldots, m_{i-1}\right\rangle+\ldots+\pi_{n}\left\langle m_{1}, m_{2}, \ldots, m_{n-1}\right\rangle, 0 \leq \pi_{i} \leq m_{i}-1, i=1,2, \ldots, n .
$$
єдиним.

Покажемо, що при всіх парних модулях таке уявлення числа в поліадичному коді не $\epsilon$

Нехай $\hat{N}=\left(\hat{\alpha}_{1}, \hat{\alpha}_{2}, \ldots, \hat{\alpha}{ }_{n}\right)$.

Виконаємо операцію $\hat{N}(i)=\hat{\alpha}_{i}-\hat{\alpha}_{1}, i=1,2, \ldots, n$.

Тоді $\hat{N}(i)$ стає кратним $m_{1}$ так само, як і $\left\langle m_{1}, m_{2}, \ldots, m_{i-1}\right\rangle$ за визначенням.

Нехай $\hat{N}(i)=\left\langle m_{1}, m_{2}, \ldots, m_{i-1}\right\rangle$. Оскільки відповідно до табл.1 найбільше значення $\pi_{i-1}=m_{i-1}-1$, a $\theta=\frac{\left\langle m_{1}, m_{2}, \ldots, m_{i-1}\right\rangle}{\left\langle m_{1}, m_{2}, \ldots, m_{i-2}\right\rangle} \leq m_{i-1}$, число $\hat{N}(i)=\left\langle m_{1}, m_{2}, \ldots, m_{i-1}\right\rangle$ може бути отримано так само, як $\pi_{i-1}\left\langle m_{1}, m_{2}, \ldots, m_{i-2}\right\rangle$ при відповідному виборі $\pi_{i-1}$. Таким чином, при всіх парних модулях представлення числа в поліадичному коді не є єдиним.

\begin{tabular}{|c|c|c|c|c|c|}
\hline$m_{1}$ & & $m_{i-1}$ & $m_{i}$ & $\ldots$ & $m_{n}$ \\
\hline $0 \leq \pi_{1} \leq m_{1}-1$ & & $m_{i-2} \leq \pi_{i-1} \leq m_{i-1}-1$ & $m_{i-1} \leq \pi_{i} \leq m_{i}-1$ & $\ldots$ & $m_{n-1} \leq \pi_{n} \leq m_{n}-1$ \\
\hline 1 & & $\pi_{i-1}\left\langle m_{1}, m_{2}, \ldots, m_{i-2}\right\rangle$ & $\pi_{i}\left\langle m_{1}, m_{2}, \ldots, m_{i-1}\right\rangle$ & $\cdots$ & $\pi_{n}\left\langle m_{1}, m_{2}, \ldots, m_{n-1}\right\rangle$ \\
\hline
\end{tabular}

\section{Таблиия 1}


Наприклад, в системі модулів $m_{1}=10, m_{2}=6, m_{3}=14, m_{4}=22 \quad$ при $\pi_{1}=0, \pi_{2}=2, \pi_{3}=3, \pi_{4}=8$ представлення числа $N=1790$ в поліадичному коді має вигляд $N=1790=0+2\langle 10\rangle+3\langle 10,6\rangle+8\langle 10,6,14\rangle$, при $\pi_{1}=0, \pi_{2}=2, \pi_{3}=10, \pi_{4}=7$ представлення того ж числа $N=1790$ в поліадичному коді має вигляд $N=1790=0+2\langle 10\rangle+10\langle 10,6\rangle+7\langle 10,6,14\rangle$.

Звідси випливає, що пошук критерію для визначення приналежності числа до першої або другої половини діапазону стає одним із напрямків дослідження СЗК з усіма парними модулями.

Реалізація операції порівняння чисел $\hat{N}_{1}=\left(\hat{\alpha}_{1}, \hat{\alpha}_{2}, \ldots, \hat{\alpha}_{n-j}\right)$ i $\hat{N}_{2}=\left(\hat{\beta}_{1}, \hat{\beta}_{2}, \ldots, \hat{\beta}_{n-j}\right)$ при всіх парних модулях по другому 3 наведених вище алгоритмів досягається таким підбором модулів, при якому $\theta_{i-1}=\frac{\left\langle m_{1}, m_{2}, \ldots, m_{i}\right\rangle}{\left\langle m_{1}, m_{2}, \ldots, m_{i-1}\right\rangle}-$ число непарне, i кожна пара чисел $\lambda_{1}=\theta_{i-1}, m_{1}, \lambda_{2}=\theta_{i-1}, m_{2}, \ldots, \lambda_{i-1}=\theta_{i-1}, m_{i-1}$ - числа взаємно прості.

Приклад порівняння пари чисел $N_{1}=532, \quad N_{2}=1828 \quad$ при $m_{1}=10, m_{2}=6, m_{3}=14, m_{4}=22$ представлений в табл.2. Тут

$$
\begin{gathered}
\theta_{1}=\frac{\left\langle m_{1}, m_{2}\right\rangle}{\left\langle m_{1}\right\rangle}=\frac{\langle 10,6\rangle}{\langle 10\rangle}=3 . \\
\theta_{2}=\frac{\left\langle m_{1}, m_{2}, m_{3}\right\rangle}{\left\langle m_{1}, m_{2}\right\rangle}=\frac{\langle 10,6,14\rangle}{\langle 10,6\rangle}=7 . \\
\theta_{3}=\frac{\left\langle m_{1}, m_{2}, m_{3}, m_{4}\right\rangle}{\left\langle m_{1}, m_{2}, m_{3}\right\rangle}=\frac{\langle 10,6,14,22\rangle}{\langle 10,6,14\rangle}=11 .
\end{gathered}
$$

\begin{tabular}{|c|c|c|c|c|c|c|c|c|}
\hline Модулі & 10 & 6 & 14 & 22 & Модулі & 10 & 6 & 14 \\
\hline $\begin{array}{c}\text { Порівнювані } \\
\text { числа }\end{array}$ & \multicolumn{4}{|c|}{ Залишки } & $\begin{array}{c}\text { Наведені } \\
\text { числа }\end{array}$ & \multicolumn{3}{|c|}{ Наведені залишки } \\
\hline & & & & & & \multicolumn{3}{|c|}{$\tilde{\alpha}_{i}^{1}=\left(\alpha_{i}-\alpha_{4}\right)\left(\bmod m_{i}\right)$} \\
\hline & & & & & & \multicolumn{3}{|c|}{$\tilde{\beta}_{i}^{1}=\left(\beta_{i}-\beta_{4}\right)\left(\bmod m_{i}\right)$} \\
\hline & $\alpha_{1}$ & $\alpha_{2}$ & $\alpha_{3}$ & $\alpha_{4}$ & & $\tilde{\alpha}_{1}^{1}$ & $\tilde{\alpha}_{2}^{1}$ & $\tilde{\alpha}_{3}^{1}$ \\
\hline & $\beta_{1}$ & $\beta_{2}$ & $\beta_{3}$ & $\beta_{4}$ & & $\tilde{\beta}_{1}^{1}$ & $\tilde{\beta}_{2}^{1}$ & $\tilde{\beta}_{3}^{1}$ \\
\hline$N_{1}^{1}=532$ & 2 & 4 & 0 & 4 & $\tilde{N}_{1}^{1}$ & 8 & 0 & 10 \\
\hline$N_{2}^{1}=1828$ & 8 & 4 & 8 & 2 & $\tilde{N}_{2}^{1}$ & 6 & 2 & 6 \\
\hline
\end{tabular}

Таблиция 2 
Оскільки $\tilde{N}_{1}^{1}(8,0,10) \neq \tilde{N}_{2}^{1}(6,2,6)$, приймаємо для другої ітерації $N_{1}^{2}=\frac{\tilde{N}_{1}^{1}}{\theta_{3}}$ і $N_{2}^{2}=\frac{\tilde{N}_{2}^{1}}{\theta_{3}}$ в якості порівнюваємих чисел.

Таблиия 3

\begin{tabular}{|c|c|c|c|c|c|c|}
\hline Модулі & 10 & 6 & 14 & Модулі & 10 & 6 \\
\hline $\begin{array}{c}\text { Порівнювані } \\
\text { числа }\end{array}$ & \multicolumn{3}{|c|}{ Залишки } & $\begin{array}{c}\text { Наведені } \\
\text { числа }\end{array}$ & \multicolumn{2}{|c|}{ Наведені залишки } \\
\hline & & & & & \multicolumn{2}{|c|}{$\tilde{\alpha}_{i}^{2}=\left(\tilde{\alpha}_{i}^{1}-\tilde{\alpha}_{3}^{1}\right)\left(\bmod m_{i}\right)$} \\
\hline & $\tilde{\alpha}_{1}^{1}$ & $\tilde{\alpha}_{2}^{1}$ & $\tilde{\alpha}_{3}^{1}$ & & $\tilde{\beta}_{i}^{2}=\left(\tilde{\beta}_{i}^{1}-\tilde{\beta}_{3}^{1}\right)\left(\bmod m_{i}\right)$ \\
\hline & $\tilde{\beta}_{1}^{1}$ & $\tilde{\beta}_{2}^{1}$ & $\tilde{\beta}_{3}^{1}$ & & $\tilde{\alpha}_{1}^{2}$ & $\tilde{\alpha}_{2}^{2}$ \\
\hline$N_{1}^{2}$ & 8 & 0 & 6 & $\tilde{N}_{1}^{2}$ & 2 & $\tilde{\beta}_{2}^{2}$ \\
\hline$N_{2}^{2}$ & 6 & 4 & 12 & $\tilde{N}_{2}^{2}$ & 4 & 0 \\
\hline
\end{tabular}

Оскільки $\tilde{N}_{1}^{2}(2,0) \neq \tilde{N}_{2}^{2}(4,4)$, приймаємо для третьої ітерації $N_{1}^{3}=\frac{\tilde{N}_{1}^{2}}{\theta_{2}}$ і $N^{3}{ }_{2}=\frac{\tilde{N}_{2}^{2}}{\theta_{2}}$ в якості порівнюваємих чисел.

Таблиия 4

\begin{tabular}{|c|c|c|c|c|c|}
\hline Модулі & 10 & 6 & Модулі & 10 \\
\hline $\begin{array}{c}\text { Порівнювані } \\
\text { числа }\end{array}$ & \multicolumn{2}{|c|}{ Залишки } & Наведені числа & Наведені залишки \\
\hline & & & & $\tilde{\alpha}_{i}^{3}=\left(\tilde{\alpha}_{i}^{2}-\tilde{\alpha}_{2}^{2}\right)\left(\bmod m_{i}\right)$ \\
\hline & & & & $\tilde{\beta}_{i}^{3}=\left(\tilde{\beta}_{i}^{2}-\tilde{\beta}_{2}^{2}\right)\left(\bmod m_{i}\right)$ \\
\hline & $\tilde{\alpha}_{1}^{2}$ & $\tilde{\alpha}_{2}^{2}$ & & $\tilde{\alpha}_{1}^{3}$ \\
\hline & $\tilde{\beta}_{1}^{2}$ & $\tilde{\beta}_{2}^{2}$ & & $\tilde{\beta}_{1}^{3}$ \\
\hline$N_{1}^{3}$ & 6 & 0 & $\tilde{N}_{1}^{3}$ & 6 \\
\hline$N_{2}^{3}$ & 2 & 4 & $\tilde{N}_{2}^{3}$ & 8 \\
\hline
\end{tabular}

Оскільки $\tilde{N}_{1}^{3}(6) \neq \tilde{N}_{2}^{3}(8)$, приймаємо для четвертої ітерації $N_{1}^{4}=\frac{\tilde{N}_{1}^{3}}{\theta_{1}}$ і $N^{4}{ }_{2}=\frac{\tilde{N}_{2}^{3}}{\theta_{1}}$ в якості порівнюваємих чисел. 
Таблиия 5

\begin{tabular}{|c|c|}
\hline Модулі & 10 \\
\hline Порівнювані числа & Залишки \\
\hline & $\tilde{\alpha}_{1}^{4}$ \\
\hline & $\tilde{\beta}_{1}^{4}$ \\
\hline$N^{4}{ }_{1}$ & 2 \\
\hline$N_{2}{ }_{2}$ & 6 \\
\hline
\end{tabular}

Оскільки $\tilde{\alpha}_{1}^{4}(2)<\tilde{\beta}_{12}^{4}(6), N_{1}(2,4,0,4)<N_{2}(8,4,8,2)$.

\section{Висновки}

Досліджено системи залишкових класів 3 попарно взаємно простими модулями i системи залишкових класів з усіма парними модулями. Показано, що представлення чисел в поліадичному коді в першому випадку є єдиним. Це дозволяє реалізувати базову проблемну операцію визначення приналежності числа до даної половині діапазону і на їі основі - операцію порівняння чисел. Показано також, що представлення чисел в другому випадку не є єдиним, в зв'язку з чим пошук рішення розглянутих вище базових проблемних операцій вимагає подальших досліджень.

\section{Список використаної літератури}

1. Полисский Ю.Д. Алгоритм табличной реализации модульного возведения в степень / /Ю.Д. Полисский // Проблеми математичного моделювання: матеріали наук.-метод. конф., 25-27 трав. 2016 р. - Дніпропетровськ. - 2016. - С. 96-100.

2. Ирхин В.П. Табличная реализация операций модулярной арифметики / В.П.Ирхин // 50 лет модулярной арифметики: тр. юбилейной Междунар. научно-техн. конф. (23.11.-25.11. 2005). - Москва: МИЭТ. - С. 268-273.

3. Методы и алгоритмы округления, масштабирования и деления чисел в модулярной арифметике / Н.И.Червяков [и др.] // 50 лет модуляр. арифметики : тр. юбилейной Междунар. научно-техн. конф. (23.11.-25.11.2005). - Москва : МИЭТ. - С. 291-310.

4. Червяков Н.И. Методы и принципы построения модулярных нейрокомпьютеров / Н.И. Червяков // 50 лет модулярной арифметики : тр. юбилейной Междунар. научно-техн. конф. (23.11.-25.11.2005). - Москва : МИЭТ. - С. 232-242.

5. Кнут,Д. Искусство программирования / Д.Кнут. - Москва : Диалектика-Вильямс, 2013. $832 \mathrm{c}$.

6. Акушский И.Я., Юдицкий Д.И. Машинная арифметика в остаточных классах. [Текст] / И.Я Акушский., Д.И. Юдицкий. - М.: Советское радио, 1968. - 440 с.

7. Полисский Ю.Д. Определение принадлежности числа, представленного системой остаточных классов, данной половине диапазона /Ю.Д.Полисский //Проблеми математичного моделювання: матеріали наук.метод. конф., 24-26 трав. 2017 м.Дніпропетровськ. - 2017. - С. 112-114.

8. Полисский Ю.Д. О некоторых подходах к выполнению проблемных операций в системе остаточных классов. [Текст] /Ю.Д.Полисский // Электронное моделирование.- 2017. - Т. 39. - № 4.- C. 105-114. 